\title{
CJEM journal club: corticosteroids use for critically ill COVID-19 patients
}

\author{
Siying S. Li $\mathrm{Li}^{1,3} \cdot$ Corinne M. Hohl $\mathbf{l}^{2,3}$
}

Received: 13 October 2020 / Accepted: 28 November 2020 / Published online: 11 January 2021

(c) Canadian Association of Emergency Physicians (CAEP)/ Association Canadienne de Médecine d'Urgence (ACMU) 2021

Keywords COVID-19 Corticosteroids $\cdot$ Steroids $\cdot$ Critical care $\cdot$ Dexamethasone $\cdot$ Methylprednisone $\cdot$ Hydrocortisone

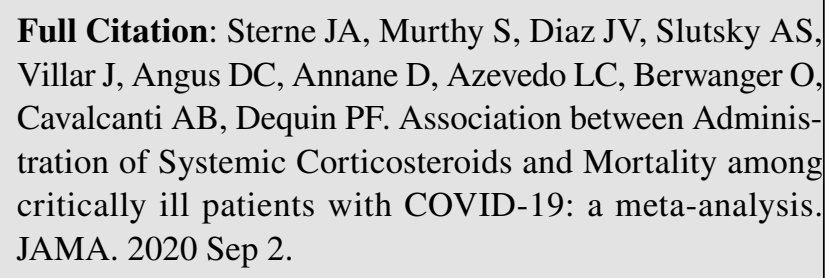

Abstract Link: https://jamanetwork.com/journals/jama/ article-abstract/2770279

Article Type:Meta-analysis

Ratings: Methods-4.5/5, Usefulness-4.5/5

\section{Introduction}

\section{Background}

The role of corticosteroids in treating critically ill COVID19 is unclear.

Siying S. Li

sili@qmed.ca

1 Royal College Emergency Medicine Training Program, University of British Columbia, Vancouver, BC, Canada

2 Centre for Clinical Epidemiology and Evaluation, Vancouver Coastal Health Research Institute, Vancouver, BC, Canada

3 Department of Emergency Medicine, University of British Columbia, Diamond Health Care Centre 11th floor, 2775 Laurel Street, Vancouver, BC V5Z 1M9, Canada

\section{Objectives}

To evaluate the effect of corticosteroid in critically ill COVID-19 patients on 28-day mortality.

\section{Methods}

\section{Design}

Prospective meta-analysis with analyses conducted prior to publication of trial data.

\section{Eligibility criteria}

Randomized controlled trials (RCTs) evaluating use of corticosteroids in critically ill COVID-19 patients compared to placebo or standard care.

\section{Outcomes}

Planned primary outcome was 30-day mortality; secondary outcome was serious adverse events.

\section{Main results}

Seven RCTs including 1703 critically ill patients were included (678 randomized to corticosteroids and 1025 to placebo or standard care). Five RCTs reported mortality at 28 days, 1 at 21 days, and 1 at 30 days. The primary analysis odds ratio (OR) for mortality was 0.66 (95\% CI $\left.0.53-0.82, P<0.001 ; I^{2}=15.6 \%, \mathrm{P}_{\text {het }}=0.31\right)$. For the 6 trials that reported serious adverse events, 64 occurred in the 354 patients randomized to corticosteroids and 80 in the 342 patients receiving placebo or standard care. Effect estimates 
for pooled dexamethasone trials and pooled hydrocortisone trials were similar. There was only one small imprecise study evaluating the effect of methylprednisone. Mechanical ventilation, duration of symptoms, and vasopressor use were not associated with increased effect of corticosteroids in exploratory subgroup analyses. GRADE assessment was reported as moderate quality.

\section{Appraisal}

\section{Strengths}

- Prospective meta-analysis of pre-publication trial data coordinated by the World Health Organization with all active trial investigators.

- RCTs were mostly low risk of bias using the Cochrane risk of bias tool 2.0. Only one study had "some concerns" for randomization process.

No significant or substantial heterogeneity across study results.

Results consistent across corticosteroid groups (dexamethasone and hydrocortisone).

Prespecified subgroup analyses.

- Multiple analysis approaches (random-effects, risk ratios) with consistent estimates.

GRADE assessment of quality of evidence performed [1].

\section{Limitations}

- Search limited to ongoing trials, which may miss unpublished negative trials.

- Small number of trials.

- RECOVERY trial contributed $57 \%$ of the weight in primary analysis [2].

- Random-effects meta-analysis of mortality was nonsignificant.

- Limited exploratory subgroup analyses due to small number of trials.

- Only one small, imprecise study evaluating effects of methylprednisone.

- Unable to meta-analyse serious adverse events due to differing definitions across trials.

The risk of harm was not clearly addressed.

\section{Context}

Following the RECOVERY trial, dexamethasone was viewed as the first RCT-proven treatment for COVID-19. However, it remained unclear whether other corticosteroids may be beneficial, which would ease access for patients. Questions also remained about whether hypoxic nonventilated patients would benefit, and if the effect may be dependent on symptom duration. This meta-analysis showed that the mortality benefit is a class effect of corticosteroids overall and found no signal for increased effect in patients receiving mechanical ventilation or in those with prolonged symptoms. Following this publication, the WHO stated that corticosteroids are now standard of care for critically ill COVID-19 patients.

\section{Bottom line}

The results of this landmark meta-analysis have made corticosteroids standard of care for critically ill COVID19 patients. Any negative trials, once published, should be included in analyses. Future research should address whether differences exist between high- versus low-dose steroids, different types of steroids, and at what point in the progression of COVID-19 does treatment with corticosteroids yield benefit. Standardization of adverse drug event documentation would also help clarify benefits versus risks of treatment [3].

\section{Reference}

1. Guyatt GH, Oxman AD, Vist GE, et al. GRADE: an emerging consensus on rating quality of evidence and strength of recommendations. BMJ. 2008;336:924-6.

2. RECOVERY Collaborative Group (2020). Dexamethasone in hospitalized patients with Covid-19-preliminary report. New Engl J Med.

3. Prescott HC, Rice TW Corticosteroids in COVID19 ARDS: Evidence and Hope During the Pandemic. JAMA. 2020;324(13):1292-1295. https://doi.org/10.1001/ jama.2020.16747 